\title{
WEIGHTED SHARING AND UNIQUENESS OF MEROMORPHIC FUNCTIONS
}

\author{
HARINA P. WAGHAMORE AND TANUJA ADAVISWAMY
}

\begin{abstract}
In this paper, we study with a weighted sharing method the uniqueness problem of $\left[f^{n} P(f)\right]^{(k)}$ and $\left[g^{n} P(g)\right]^{(k)}$ sharing one value and obtain some results which extend and improve the results due to Hong-Yan $\mathrm{Xu}$ and Ting-Bin Cao.
\end{abstract}

\section{Introduction}

Let $f$ be a non-constant meromorphic function in the whole complex plane. We shall use the following standard notations of the value distribution theory:

$$
T(r, f), m(r, f), N(r, f), \bar{N}(r, f), \ldots
$$

(See Hayman [3], Yang [6] and Yi and Yang [7]). We denote by $S(r, f)$ any quantity satisfying $S(r, f)=o(T(r, f))$

as $r \rightarrow+\infty$, possibly outside of a set with finite measure. For any constant ${ }^{\prime} a^{\prime}$, we define

$$
\Theta(a, f)=1-\limsup _{r \rightarrow \infty} \frac{\bar{N}\left(r, \frac{1}{(f-a)}\right)}{T(r, f)},
$$

Let ${ }^{\prime} a^{\prime}$ be a finite complex number and $k$ a positive integer. We denote by $N_{k)}\left(r, \frac{1}{(f-a)}\right)$ the counting function for the zeros of $f(z)-a$ with the multiplicity $\leq k$, and by $\bar{N}_{k)}\left(r, \frac{1}{(f-a)}\right)$ the corresponding one for which the multiplicity is not counted. Let $N_{(k}\left(r, \frac{1}{(f-a)}\right)$ be the counting function for the zeros of $f(z)-a$ with multiplicity atleast $k$, and $\bar{N}_{(k}\left(r, \frac{1}{(f-a)}\right)$ be the corresponding one for which the multiplicity is not counted. Set

$$
N_{k}\left(r, \frac{1}{(f-a)}\right)=\bar{N}\left(r, \frac{1}{(f-a)}\right)+\bar{N}_{(2}\left(r, \frac{1}{(f-a)}\right)+\cdots+\bar{N}_{(k}\left(r, \frac{1}{(f-a)}\right) .
$$

Received May 26, 2011, accepted September 11, 2013. 2010 Mathematics Subject Classification. Primary 30D35.

Key words and phrases. Weighted sharing, entire functions, meromorphic functions, differential polynomials, uniqueness.

Corresponding author: Harina P. Waghamore. 
We define

$$
\delta_{k}(a, f)=1-\limsup _{r \rightarrow \infty} \frac{\bar{N}_{k}\left(r, \frac{1}{(f-a)}\right)}{T(r, f)} .
$$

Let $g$ be a meromorphic function. If $f(z)-a$ and $g(z)-a$, assume the same zeros with the same multiplicities then we say that $f(z)$ and $g(z)$ share the value ' $a$ ' CM, where ' $a^{\prime}$ is a complex number. Similarly, we say that $f$ and $g$ share $a \mathrm{IM}$, provided that $f(z)-a$ and $g(z)-a$ have same multiplicities.

In 1996, Fang proved the following result.

Theorem A([1]). Let $f$ and $g$ be two non-constant entire functions and let $n, k$ be two positive integers with $n>2 k+4$. If $\left[f^{n}\right]^{(k)}$ and $\left[g^{n}\right]^{(k)}$ share the value $1 C M$, then either $f(z)=c_{1} e^{c z}$ and $g(z)=c_{2} e^{-c z}$ where $c_{1}, c_{2}$ and $c$ are three constants satisfying $(-1)^{k}\left(c_{1} c_{2}\right)^{n}(n c)^{2 k}=1$ or $f=\operatorname{tg}$ for a constant $t$ such that $t^{n}=1$.

In 1997, Yang and Hua obtained a unicity theorem corresponding to above result.

Theorem $\mathbf{B}([8])$. Let $f$ and $g$ be two nonconstant entire functions, $n \geq 6$ a positive integer. If $f^{n} f^{\prime}$ and $g^{n} g^{\prime}$ share $1 C M$, then either $f(z)=c_{1} e^{c z}$ and $g(z)=c_{2} e^{-c z}$ where $c_{1}, c_{2}$ and c are three constants satisfying $\left(c_{1} c_{2}\right)^{n+1} c^{2}=1$ or $f=\operatorname{tg}$ for a constant $t$ such that $t^{n+1}=1$.

In 2002, Fang proved the following result.

Theorem C([2]). Let $f$ and $g$ be two non-constant entire functions and let $n, k$ be two positive integers with $n>2 k+8$. If $\left[f^{n}(f-1)\right]^{(k)}$ and $\left[g^{n}(g-1)\right]^{(k)}$ share the value $1 C M$, then $f \equiv g$.

In 2008, Zhang and Lin, Zhang, Chen and Lin extended Theorem C and obtain the following results.

Theorem $\mathbf{D}([10])$. Let $f$ and $g$ be two non-constant entire functions and let $n, m$ and $k$ be three positive integers with $n>2 k+m+4$, and $\lambda$, $\mu$ be constants such that $|\lambda|+|\mu| \neq 0$. If $\left[f^{n}\left(\mu f^{m}+\lambda\right)\right]^{(k)}$ and $\left[g^{n}\left(\mu g^{m}+\lambda\right)\right]^{(k)}$ share 1 CM, then

(i) when $\lambda \mu \neq 0, f \equiv g$.

(ii) when $\lambda \mu=0$, either $f \equiv t g$, where $t$ is a constant satisfying $t^{n+m}=1$, or $f(z)=c_{1} e^{c z}$ and $g(z)=c_{2} e^{-c z}$ where $c_{1}, c_{2}$ and c are three constants satisfying $(-1)^{k} \lambda^{2}\left(c_{1} c_{2}\right)^{n+m}[(n+$ $m) c]^{2 k}=1$ or $(-1)^{k} \mu^{2}\left(c_{1} c_{2}\right)^{n+m}[(n+m) c]^{2 k}=1$.

Theorem $\mathbf{E}([11])$. Let $f$ and $g$ be two non-constant entire functions and let $n, m$ and $k$ be three positive integers with $n>2 k+m+4$, and let $P(z)=a_{m} z^{m}+a_{m-1} z^{m-1}+\cdots+a_{1} z+a_{0}$ or $P(z) \equiv c_{0}$, where $a_{0} \neq 0, a_{1}, \ldots, a_{m-1}, a_{m} \neq 0, c_{0} \neq 0$ are complex constants. If $\left[f^{n} P(f)\right]^{(k)}$ and $\left[g^{n} P(g)\right]^{(k)}$ share 1 CM, then 
(i) when $P(z)=a_{m} z^{m}+a_{m-1} z^{m-1}+\cdots+a_{1} z+a_{0}$, either $f \equiv$ tg for a constant $t$ such that $t^{d}=1$, where $d=(n+m, \ldots, n+m-i, \ldots, n), a_{m-i} \neq 0$ for some $i=0,1, \ldots, m$, or $f$ and $g$ satisfy the algebraic equation $R(f, g) \equiv 0$, where $R\left(\omega_{1}, \omega_{2}\right)=\omega_{1}^{n}\left(a_{m} \omega_{1}^{m}+a_{m-1} \omega_{1}^{m-1}+\cdots+\right.$ $\left.a_{1} \omega_{1}+a_{0}\right)-\omega_{2}^{n}\left(a_{m} \omega_{2}^{m}+a_{m-1} \omega_{2}^{m-1}+\cdots+a_{1} \omega_{2}+a_{0}\right) ;$

(ii) when $P(z)=c_{0}$, either $f(z)=c_{1} / \sqrt[n]{c_{0}} e^{c z}, g(z)=c_{2} / \sqrt[n]{c_{0}} e^{-c z}$, where $c_{1}, c_{2}$ and c are three constants satisfying $(-1)^{k}\left(c_{1} c_{2}\right)^{n}(n c)^{2 k}=1$, or $f=\operatorname{tg}$ for a constant $t$ such that $t^{n}=1$.

In 2009, H.-Y. Xu and T.-B. Cao proved the following result.

Theorem $\mathbf{F}([5])$. Let $f$ and $g$ be two nonconstant entire functions, and let $n, m$ and $k$ be three positive integers with $n \geq 5 k+5 m+8$. If $\left[f^{n} P(f)\right]^{(k)}$ and $\left[g^{n} P(g)\right]^{(k)}$ share $(1,0)$, then the conclusion of Theorem E still holds.

Theorem G([5]). Let $f$ and $g$ be two nonconstant entire functions, and let $n, m$ and $k$ be three positive integers with $n>\frac{9}{2} m+4 k+\frac{9}{2}$. If $\left[f^{n} P(f)\right]^{(k)}$ and $\left[g^{n} P(g)\right]^{(k)}$ share $(1,1)$, then the conclusion of Theorem E still holds.

Theorem $\mathbf{H}([5])$. Let $f$ and $g$ be two nonconstant entire functions, and let $n, m$ and $k$ be three positive integers with $n \geq 3 m+3 k+5$. If $\left[f^{n} P(f)\right]^{(k)}$ and $\left[g^{n} P(g)\right]^{(k)}$ share $(1,2)$, then the conclusion of Theorem Estill holds.

In this paper, by introducing the notion of multiplicity, we reduce and improve Theorems F, G and $\mathrm{H}$. Also we extend these theorems to meromorphic functions and obtain the following results.

Theorem 1.1. Let $f$ and $g$ be two non-constant meromorphic functions, whose zeros and poles are of multiplicities atleast $s$, where $s$ is a positive integer. Let $P(f)=a_{m} f^{m}+a_{m-1} f^{m-1}+\cdots+$ $a_{1} f+a_{0},\left(a_{m} \neq 0\right)$, and $a_{i}(i=0,1, \ldots, m)$ is the first nonzero coefficient from the right, and let $n, k, m$ be three positive integers. If $\left[f^{n} P(f)\right]^{(k)}$ and $\left[g^{n} P(g)\right]^{(k)}$ share $(1, l)$ and one of the following conditions holds:

(i) $l \geq 2$ and $s(n+m)>3 k+10$

(ii) $l=1$ and $s(n+m)>5 k+13$

(iii) $l=0$ and $s(n+m)>9 k+16$

then either $f=\operatorname{tg}$ for a constant $t$ such that $t^{d}=1$, where $d=(n+m, \ldots, n+m-i, \ldots, n)$, $a_{m-i} \neq 0$ for some $i=0,1, \ldots, m$, or $f$ and $g$ satisfy the algebraic equation $R(f, g) \equiv 0$, where $R\left(\omega_{1}, \omega_{2}\right)=\omega_{1}^{n} P\left(\omega_{1}\right)-\omega_{2}^{n} P\left(\omega_{2}\right)$

Theorem 1.2. Let $f$ and $g$ be two non-constant entire functions, whose zeros and poles are of multiplicities atleast s, where sis a positive integer. Let $P(f)=a_{m} f^{m}+a_{m-1} f^{m-1}+\cdots+a_{1} f+a_{0}$, 
$\left(a_{m} \neq 0\right)$, and $a_{i}(i=0,1, \ldots, m)$ is the first nonzero coefficient from the right, and let $n, k, m$ be three positive integers. If $\left[f^{n} P(f)\right]^{(k)}$ and $\left[g^{n} P(g)\right]^{(k)}$ share $(1, l)$ and one of the following conditions holds:

(i) $l \geq 2$ and $s(n+m)>3 k+5$

(ii) $l=1$ and $s(n+m)>4 k+6$

(iii) $l=0$ and $s(n+m)>5 k+8$

then either $f=\operatorname{tg}$ for a constant $t$ such that $t^{d}=1$, where $d=(n+m, \ldots, n+m-i, \ldots, n)$, $a_{m-i} \neq 0$ for some $i=0,1, \ldots, m$, or $f$ and $g$ satisfy the algebraic equation $R(f, g) \equiv 0$, where $R\left(\omega_{1}, \omega_{2}\right)=\omega_{1}^{n} P\left(\omega_{1}\right)-\omega_{2}^{n} P\left(\omega_{2}\right)$.

Remark. In Theorem 1.2, giving specific values for $s$, we get the following interesting cases:

(i) If $s=1$, then for $l \geq 2$ we get $n>3 k+5-m$, for $l=1$ we get $n>4 k+6-m$ and for $l=0$ we get $n>5 k+8-m$.

(ii) If $s=2$, then for $l \geq 2$ we get $n>\frac{3 k+5}{2}-m$, for $l=1$ we get $n>2 k+3-m$ and for $l=0$ we get $n>\frac{5 k+8}{2}-m$.

We conclude that if $f$ and $g$ have zeros and poles of higher order multiplicity, then we can reduce the value of $n$.

\section{Some Lemmas}

Lemma 2.1 ([3]). Let $f$ be a nonconstant meromorphic function, let $k$ be a positive integer, and let c be a nonzero finite complex number. Then

$$
\begin{aligned}
T(r, f) & \leq \bar{N}(r, f)+N\left(r, \frac{1}{f}\right)+N\left(r, \frac{1}{f^{(k)}-c}\right)-N\left(r, \frac{1}{f^{(k+1)}}\right)+S(r, f) \\
& \leq \bar{N}(r, f)+N_{k+1}\left(r, \frac{1}{f}\right)+\bar{N}\left(r, \frac{1}{f^{(k)}-c}\right)-N_{0}\left(r, \frac{1}{f^{(k+1)}}\right)+S(r, f) .
\end{aligned}
$$

where $N_{0}\left(r, \frac{1}{f^{(k+1)}}\right)$ is the counting function which only counts those points such that $f^{(k+1)}=0$ but $f\left(f^{(k)}-c\right) \neq 0$.

Lemma 2.2 ([9]). Let $f$ be a nonconstant meromorphic function and $P(f)=a_{0}+a_{1} f+\cdots+$ $a_{n} f^{n}$, where $a_{0}, a_{1}, \ldots, a_{n}$ are constants and $a_{n} \neq 0$. Then

$$
T(r, P(f))=n T(r, f)+S(r, f)
$$


Lemma 2.3 ([4, 12]). Let $f$ be a non-constant meromorphic function and $k$ be a positive integer, then

$$
\begin{aligned}
N_{p}\left(r, \frac{1}{f^{(k)}}\right) & \leq N_{p+k}\left(r, \frac{1}{f}\right)+k \bar{N}(r, f)+S(r, f) \\
& \leq(p+k) \bar{N}\left(r, \frac{1}{f}\right)+k \bar{N}(r, f)+S(r, f) .
\end{aligned}
$$

This Lemma can be obtained immediately from the proof of Lemma 2.3 in [4] which is the case $p=2$.

Lemma 2.4 ([13]). Let $F$ and $G$ be two nonconstant meromorphic functions. If $F$ and $G$ share $1 \mathrm{IM}$, then $\bar{N}_{L}\left(r, \frac{1}{F-1}\right) \leq \bar{N}\left(r, \frac{1}{F}\right)+\bar{N}(r, F)+S(r, F)$.

Lemma 2.5 ([5]). Let $f$ and $g$ be two nonconstant entire functions, and let $k$ be a positive integer. If $f^{(k)}$ and $g^{(k)}$ share $(1, l)(l=0,1,2)$. Then

(i) If $l=0$,

$\Theta(0, f)+\delta_{k}(0, f)+\delta_{k+1}(0, f)+\delta_{k+1}(0, g)+\delta_{k+2}(0, f)+\delta_{k+2}(0, g)>5$, then either $f^{(k)} g^{(k)}=$ 1 or $f \equiv g$;

(ii) If $l=1$,

$\frac{1}{2}\left[\Theta(0, f)+\delta_{k}(0, f)+\delta_{k+2}(0, f)\right]+\delta_{k+1}(0, f)+\delta_{k+1}(0, g)+\Theta(0, g)+\delta_{k}(0, g)>\frac{9}{2}$, then either $f^{(k)} g^{(k)}=1$ or $f \equiv g$;

(iii) If $l=2$,

$\Theta(0, f)+\delta_{k}(0, f)+\delta_{k+1}(0, f)+\delta_{k+2}(0, g)>3$, then either $f^{(k)} g^{(k)}=1$ or $f \equiv g$.

Lemma 2.6. Let $f$ and $g$ be two nonconstant meromorphic functions, $k(\geq 1)$ and $l(\geq 0)$ be integers. If $f^{(k)}$ and $g^{(k)}$ share $(1, l)(l=0,1,2)$. Then

(i) If $l \geq 2$,

$(k+2) \Theta(\infty, f)+2 \Theta(\infty, g)+\Theta(0, f)+\Theta(0, g)+\delta_{k+1}(0, f)+\delta_{k+1}(0, g)>k+7$, then either $f^{(k)} g^{(k)}=1$ or $f \equiv g$;

(ii) If $l=1$,

$(2 k+3) \Theta(\infty, f)+2 \Theta(\infty, g)+\Theta(0, f)+\Theta(0, g)+\delta_{k+1}(0, f)+\delta_{k+1}(0, g)+\delta_{k+2}(0, f)>2 k+9$ then either $f^{(k)} g^{(k)}=1$ or $f \equiv g$;

(iii) If $l=0$,

$(2 k+3) \Theta(\infty, f)+(2 k+4) \Theta(\infty, g)+\Theta(0, f)+\Theta(0, g)+2 \delta_{k+1}(0, f)+3 \delta_{k+1}(0, g)>4 k+13$ then either $f^{(k)} g^{(k)}=1$ or $f \equiv g$.

Proof. Let

$$
\Phi(z)=\left(\frac{f^{(k+2)}}{f^{(k+1)}}-2 \frac{f^{(k+1)}}{f^{(k)}-1}\right)-\left(\frac{g^{(k+2)}}{g^{(k+1)}}-2 \frac{g^{(k+1)}}{g^{(k)}-1}\right)
$$


Suppose that $\Phi(z) \neq 0$. If $z_{0}$ is a common simple 1-point of $f^{(k)}(z)$ and $f^{(k)}(z)$, substituting their Taylor series at $z_{0}$ into (2.1), we can get $\Phi\left(z_{0}\right)=0$. Thus we have,

$$
\begin{aligned}
N_{E}^{1)}\left(r, \frac{1}{f^{(k)}-1}\right)=N_{E}^{1)}\left(r, \frac{1}{g^{(k)}-1}\right) & \leq \bar{N}\left(r, \frac{1}{\Phi}\right) \leq T(r, \Phi)+O(1) \\
& \leq N(r, \Phi)+S(r, f)+S(r, g),
\end{aligned}
$$

where $N_{E}^{1)}\left(r, \frac{1}{f^{(k)}-1}\right)$ denotes the counting function of common 1-points of $f^{(k)}$ and $g^{(k)}$.

According to our assumption, $\Phi(z)$ has simple poles only at zeros of $f^{(k+1)}, f^{(k)}-1$ and $g^{(k+1)}, g^{(k)}-1$ as well as poles of $f$ and $g$.

From Lemma 2.1, we have

$$
\begin{aligned}
T(r, f)+T(r, g) \leq & \bar{N}(r, f)+\bar{N}(r, g)+N_{k+1}\left(r, \frac{1}{f}\right)+N_{k+1}\left(r, \frac{1}{g}\right) \\
& +\bar{N}\left(r, \frac{1}{f^{(k)}-1}\right)+\bar{N}\left(r, \frac{1}{g^{(k)}-1}\right)-N_{0}\left(r, \frac{1}{f^{(k+1)}}\right) \\
& -N_{0}\left(r, \frac{1}{g^{(k+1)}}\right)+S(r, f)+S(r, g) .
\end{aligned}
$$

Obviously,

$$
N\left(r, \frac{1}{f^{(k)}-1}\right) \leq T\left(r, f^{(k)}\right)+0(1) \leq T(r, f)+k \bar{N}(r, f)+S(r, f) .
$$

If $l \geq 2$, we have

$$
\begin{aligned}
N(r, \Phi) \leq & \bar{N}(r, f)+\bar{N}\left(r, \frac{1}{f}\right)+\bar{N}(r, g)+\bar{N}\left(r, \frac{1}{g}\right)+\bar{N}_{(l+1}\left(r, \frac{1}{f^{(k)}-1}\right) \\
& +N_{0}\left(r, \frac{1}{f^{(k+1)}}\right)+N_{0}\left(r, \frac{1}{g^{(k+1)}}\right),
\end{aligned}
$$

and

$$
\begin{aligned}
& \bar{N}_{(l+1}\left(r, \frac{1}{f^{(k)}-1}\right)+\bar{N}\left(r, \frac{1}{f^{(k)}-1}\right)+\bar{N}\left(r, \frac{1}{g^{(k)}-1}\right) \\
& \quad \leq N_{1)}\left(r, \frac{1}{g^{(k)}-1}\right)+N\left(r, \frac{1}{f^{(k)}-1}\right) .
\end{aligned}
$$

From (2.2)-(2.6) we deduce that

$$
\begin{aligned}
T(r, g) & \leq(k+2) \bar{N}(r, f)+2 \bar{N}(r, g)+\bar{N}\left(r, \frac{1}{f}\right)+\bar{N}\left(r, \frac{1}{g}\right)+N_{k+1}\left(r, \frac{1}{f}\right) \\
& +N_{k+1}\left(r, \frac{1}{g}\right)+S(r, f)+S(r, g) .
\end{aligned}
$$

Without loss of generality, we suppose that there exists a set I with infinite linear measure such that $T(r, F) \leq T(r, G)$ for $r \in I$. Hence

$$
T(r, g) \leq[(k+2)(1-\Theta(\infty, f))+2(1-\Theta(\infty, g))+(1-\Theta(0, f))
$$




$$
\left.+(1-\Theta(0, g))+\left(1-\delta_{k+1}(0, f)\right)+\left(1-\delta_{k+1}(0, g)\right)+\varepsilon\right] T(r, g)+S(r, g),
$$

for $r \in I$ and $0<\varepsilon<\Delta_{1}-(k+7)$, that is $\left[\Delta_{1}-(k+7)-\varepsilon\right] T(r, g) \leq S(r, g)$.

ie.,

$$
\Delta_{1} \leq(k+7)
$$

If $l=1$, then

$$
\begin{aligned}
N(r, \Phi) \leq & \bar{N}(r, f)+\bar{N}\left(r, \frac{1}{f}\right)+\bar{N}(r, g)+\bar{N}\left(r, \frac{1}{g}\right)+\bar{N}_{(2}\left(r, \frac{1}{f^{(k)}-1}\right) \\
& +N_{0}\left(r, \frac{1}{f^{(k+1)}}\right)+N_{0}\left(r, \frac{1}{g^{(k+1)}}\right) .
\end{aligned}
$$

Obviously,

$$
\bar{N}\left(r, \frac{1}{f^{(k)}-1}\right)+\bar{N}\left(r, \frac{1}{g^{(k)}-1}\right) \leq N_{E}^{1)}\left(r, \frac{1}{f^{(k)}-1}\right)+N\left(r, \frac{1}{f^{(k)}-1}\right) .
$$

Thus, we deduce from (2.2)-(2.4), (2.8) and (2.9) that

$$
\begin{aligned}
T(r, g) \leq & (k+2) \bar{N}(r, f)+2 \bar{N}(r, g)+\bar{N}\left(r, \frac{1}{f}\right)+\bar{N}\left(r, \frac{1}{g}\right)+N_{k+1}\left(r, \frac{1}{f}\right) \\
& +N_{k+1}\left(r, \frac{1}{g}\right)+\bar{N}_{(2}\left(r, \frac{1}{f^{(k)}-1}\right)+S(r, f)+S(r, g) .
\end{aligned}
$$

Note that $l=1$, from Lemma 2.3, we have

$$
\begin{aligned}
\bar{N}_{(2}\left(r, \frac{1}{f^{(k)}-1}\right) & \leq \bar{N}\left(r, \frac{1}{f^{(k+1)}}\right)=N_{1}\left(r, \frac{1}{f^{(k+1)}}\right) \\
& \leq N_{k+2}\left(r, \frac{1}{f}\right)+(k+1) \bar{N}(r, f)+S(r, f) .
\end{aligned}
$$

The inequality (2.10) together with (2.11) yields

$$
\begin{aligned}
T(r, g) \leq & (2 k+3) \bar{N}(r, f)+2 \bar{N}(r, g)+\bar{N}\left(r, \frac{1}{f}\right)+\bar{N}\left(r, \frac{1}{g}\right)+N_{k+1}\left(r, \frac{1}{f}\right) \\
& +N_{k+1}\left(r, \frac{1}{g}\right)+N_{k+2}\left(r, \frac{1}{f}\right)+S(r, f)+S(r, g) .
\end{aligned}
$$

Hence

$$
\begin{aligned}
T(r, g) \leq & {[(2 k+3)(1-\Theta(\infty, f))+2(1-\Theta(\infty, g))+(1-\Theta(0, f))} \\
& +(1-\Theta(0, g))+\left(1-\delta_{k+1}(0, f)\right)+\left(1-\delta_{k+1}(0, g)\right)+\left(1-\delta_{k+2}(0, f)\right) \\
& +\varepsilon] T(r, g)+S(r, g),
\end{aligned}
$$

for $r \in I$ and $0<\varepsilon<\Delta_{2}-(2 k+9)$, that is $\left[\Delta_{2}-(2 k+9)-\varepsilon\right] T(r, g) \leq S(r, g)$, ie.,

$$
\Delta_{2} \leq(2 k+9)
$$


If $l=0$, i.e., $f^{(k)}$ and $g^{(k)}$ share $1 \mathrm{IM}$, at this circumstance, we have

$$
\begin{aligned}
N(r, \Phi) \leq & \bar{N}(r, f)+\bar{N}\left(r, \frac{1}{f}\right)+\bar{N}(r, g)+\bar{N}\left(r, \frac{1}{g}\right)+\bar{N}_{L}\left(r, \frac{1}{f^{(k)}-1}\right) \\
& +\bar{N}_{L}\left(r, \frac{1}{g^{(k)}-1}\right)+N_{0}\left(r, \frac{1}{f^{(k+1)}}\right)+N_{0}\left(r, \frac{1}{g^{(k+1)}}\right) .
\end{aligned}
$$

From Lemma 2.4, we have

$$
\begin{aligned}
\bar{N}_{L}\left(r, \frac{1}{f^{(k)}-1}\right)+2 \bar{N}_{L}\left(r, \frac{1}{g^{(k)}-1}\right) \leq & \bar{N}(r, f)+2 \bar{N}(r, g)+\bar{N}\left(r, \frac{1}{f^{(k)}}\right) \\
& +2 \bar{N}\left(r, \frac{1}{g^{(k)}}\right)+S(r, f)+S(r, g) .
\end{aligned}
$$

From Lemma 2.3, we can deduce that

$$
\begin{aligned}
& \bar{N}\left(r, \frac{1}{f^{(k)}}\right)+2 \bar{N}\left(r, \frac{1}{g^{(k)}}\right)=N_{1}\left(r, \frac{1}{f^{(k)}}\right)+2 N_{1}\left(r, \frac{1}{g^{(k)}}\right) \\
& \quad \leq N_{k+1}\left(r, \frac{1}{f}\right)+2 N_{k+1}\left(r, \frac{1}{g}\right)+k \bar{N}(r, f)+2 k \bar{N}(r, g)+S(r, f)+S(r, g) .
\end{aligned}
$$

When $l=0$, we can get

$$
\bar{N}\left(r, \frac{1}{f^{(k)}-1}\right)+\bar{N}\left(r, \frac{1}{g^{(k)}-1}\right) \leq N_{E}^{1)}\left(r, \frac{1}{f^{(k)}-1}\right)+\bar{N}_{L}\left(r, \frac{1}{g^{(k)}-1}\right)+N\left(r, \frac{1}{f^{(k)}-1}\right) .
$$

From (2.2) - (2.4) and (2.13) - (2.15) and the above inequality, we can obtain

$$
\begin{aligned}
T(r, g) \leq & (2 k+3) \bar{N}(r, f)+(2 k+4) \bar{N}(r, g)+\bar{N}\left(r, \frac{1}{f}\right)+\bar{N}\left(r, \frac{1}{g}\right) \\
& +2 N_{k+1}\left(r, \frac{1}{f}\right)+3 N_{k+1}\left(r, \frac{1}{g}\right)+S(r, f)+S(r, g) .
\end{aligned}
$$

In the same way, we can also get

$$
\begin{aligned}
T(r, g) \leq & {[(2 k+3)(1-\Theta(\infty, f))+(2 k+4)(1-\Theta(\infty, g))+(1-\Theta(0, f))} \\
& \left.+(1-\Theta(0, g))+2\left(1-\delta_{k+1}(0, f)\right)+3\left(1-\delta_{k+1}(0, g)\right)+\varepsilon\right] T(r, g)+S(r, g),
\end{aligned}
$$

for $r \in I$ and $0<\varepsilon<\Delta_{3}-(4 k+13)$, that is $\left[\Delta_{3}-(4 k+13)-\varepsilon\right] T(r, g) \leq S(r, g)$, ie.,

$$
\Delta_{3} \leq(4 k+13)
$$

Hence, we get $\Phi(z) \equiv 0$, ie.,

$$
\frac{f^{(k+2)}}{f^{(k+1)}}-2 \frac{f^{(k+1)}}{f^{(k)}-1}=\frac{g^{(k+2)}}{g^{(k+1)}}-2 \frac{g^{(k+1)}}{g^{(k)}-1} .
$$

Integration yields

$$
\frac{1}{f^{(k)}-1} \equiv \frac{b g^{(k)}+a-b}{g^{(k)}-1},
$$

where $a$ and $b$ are two constants and $a \neq 0$. By using the same argument as in [13], we can obtain $f^{(k)} g^{(k)} \equiv 1$ or $f \equiv g$, we here omit the detail. The proof of Lemma 2.6 is completed. 
Lemma 2.7. Let $f$ and $g$ be two non-constant meromorphic functions, and let $n(\geq 1), k(\geq 1)$ and $m(\geq 1)$ be a integers. Then

$$
\left[f^{n} P(f)\right]^{(k)}\left[g^{n} P(g)\right]^{(k)} \neq 1 .
$$

Proof. Let

$$
\left[f^{n} P(f)\right]^{(k)}\left[g^{n} P(g)\right]^{(k)} \equiv 1 .
$$

Let $z_{0}$ be a zero of $f$ of order $p_{0}$. From (2.18) we get $z_{0}$ is a pole of $g$. Suppose that $z_{0}$ is a pole of $g$ of order $q_{0}$. Again by (2.18), we obtain $n p_{0}-k=n q_{0}+m q_{0}+k$,

i.e., $n\left(p_{0}-q_{0}\right)=m q_{0}+2 k$.

which implies that $q_{0} \geq \frac{n-2 k}{m}$ and so we have $p_{0} \geq \frac{n+m-2 k}{m}$.

Let $z_{1}$ be a zero of $f-1$ of order $p_{1}$, then $z_{1}$ is a zero of $\left[f^{n} P(f)\right]^{(k)}$ of order $p_{1}-k$. Therefore from (2.18) we obtain $p_{1}-k=n q_{1}+m q_{1}+k$

i.e., $p_{1} \geq(n+m) s+2 k$.

Let $z_{2}$ be a zero of $f^{\prime}$ of order $p_{2}$ that is not a zero of $f P(f)$, then from (2.18) $z_{2}$ is a pole of $g$ of order $q_{2}$. Again by (2.18) we get $p_{2}-(k-1)=n q_{2}+m q_{2}+k$

i.e., $p_{2} \geq(n+m) s+2 k-1$.

In the same manner as above, we have similar results for the zeros of $\left[g^{n} P(g)\right]^{(k)}$.

On other hand, suppose that $z_{3}$ is a pole of $f$. From (2.18), we get that $z_{3}$ is the zero of $\left[g^{n} P(g)\right]^{(k)}$.

Thus

$$
\begin{aligned}
\bar{N}(r, f) & \leq \bar{N}\left(r, \frac{1}{g}\right)+\bar{N}\left(r, \frac{1}{g-1}\right)+\bar{N}\left(r, \frac{1}{g^{\prime}}\right) \\
& \leq \frac{1}{p_{0}} N\left(r, \frac{1}{g}\right)+\frac{1}{p_{1}} N\left(r, \frac{1}{g-1}\right)+\frac{1}{p_{2}} N\left(r, \frac{1}{g^{\prime}}\right) \\
& \leq\left[\frac{m}{n+m-2 k}+\frac{1}{(n+m) s+2 k}+\frac{2}{(n+m) s+2 k-1}\right] T(r, g)+S(r, g) .
\end{aligned}
$$

By second fundamental theorem and equation (2.19), we have

$$
\begin{aligned}
T(r, f) \leq & \bar{N}\left(r, \frac{1}{f}\right)+\bar{N}\left(r, \frac{1}{f-1}\right)+\bar{N}(r, f) \\
\leq & \frac{m}{n+m-2 k} N\left(r, \frac{1}{f}\right)+\frac{1}{(n+m) s+2 k} N\left(r, \frac{1}{f-1}\right) \\
& +\left[\frac{m}{n+m-2 k}+\frac{1}{(n+m) s+2 k}+\frac{2}{(n+m) s+2 k-1}\right] T(r, g)+S(r, g)+S(r, f) . \\
T(r, f) \leq & {\left[\frac{m}{n+m-2 k}+\frac{1}{(n+m) s+2 k}\right] T(r, f) }
\end{aligned}
$$




$$
+\left[\frac{m}{n+m-2 k}+\frac{1}{(n+m) s+2 k}+\frac{2}{(n+m) s+2 k-1}\right] T(r, g)+S(r, g)+S(r, f) .
$$

Similarly, we have

$$
\begin{aligned}
T(r, g) \leq & {\left[\frac{m}{n+m-2 k}+\frac{1}{(n+m) s+2 k}\right] T(r, g) } \\
& +\left[\frac{m}{n+m-2 k}+\frac{1}{(n+m) s+2 k}+\frac{2}{(n+m) s+2 k-1}\right] T(r, f)+S(r, g)+S(r, f) .
\end{aligned}
$$

Adding (2.20) and (2.21) we get

$$
\begin{aligned}
T(r, f)+T(r, g) \leq & {\left[\frac{2 m}{n+m-2 k}+\frac{2}{(n+m) s+2 k}+\frac{2}{(n+m) s+2 k-1}\right]\{T(r, f)+T(r, g)\} } \\
& +S(r, g)+S(r, f) .
\end{aligned}
$$

which is a contradiction. Thus Lemma proved.

\section{Proofs of the Theorems}

In this section we present the proofs of the main results.

Proof of Theorem 1.1. Let $F=f^{n} P(f)$ and $G=g^{n} P(g)$.

Consider

$$
\begin{gathered}
\bar{N}\left(r, \frac{1}{F}\right)=\bar{N}\left(r, \frac{1}{f^{n} P(f)}\right) \leq \frac{1}{s(n+m)} N\left(r, \frac{1}{F}\right) \leq \frac{2}{s(n+m)}[T(r, F)+O(1)] . \\
\Theta(0, F)=1-\limsup _{r \rightarrow \infty} \frac{\bar{N}\left(r, \frac{1}{F}\right)}{T(r, F)} \geq 1-\frac{2}{s(n+m)} .
\end{gathered}
$$

Similarly,

$$
\begin{gathered}
\Theta(0, G) \geq 1-\frac{2}{s(n+m)} . \\
\Theta(\infty, F)=1-\limsup _{r \rightarrow \infty} \frac{\bar{N}(r, F)}{T(r, F)} \geq 1-\frac{1}{s(n+m)} .
\end{gathered}
$$

Similarly,

$$
\Theta(\infty, G) \geq 1-\frac{1}{s(n+m)} .
$$

Consider

$$
N_{k+1}\left(r, \frac{1}{F}\right)=N_{k+1}\left(r, \frac{1}{f^{n} P(f)}\right)=(k+1) \bar{N}\left(r, \frac{1}{f^{n} P(f)}\right) \leq \frac{(k+1)}{s(n+m)}[T(r, F)+O(1)] .
$$

Next, we have

$$
\delta_{k+1}(0, F)=1-\limsup _{r \rightarrow \infty} \frac{N_{k+1}\left(r, \frac{1}{F}\right)}{T(r, F)} \geq 1-\frac{(k+1)}{s(n+m)} .
$$


Similarly,

$$
\delta_{k+1}(0, G) \geq 1-\frac{(k+1)}{s(n+m)} .
$$

Case(i) If $l \geq 2$ and from (3.1) to (3.6) and also from Lemma 2.6, we get

$$
\begin{aligned}
\Delta_{1} & =(k+2) \Theta(\infty, f)+2 \Theta(\infty, g)+\Theta(0, f)+\Theta(0, g)+\delta_{k+1}(0, f)+\delta_{k+1}(0, g) \\
& >(k+8)-\frac{3 k+10}{s(n+m)}
\end{aligned}
$$

Since $s(n+m)>3 k+10$, we get $\Delta_{1}>k+7$.

Therefore, by Lemma 2.6, we deduce that either $F^{(k)} G^{(k)} \equiv 1$ or $F \equiv G$.

If $F^{(k)} G^{(k)} \equiv 1$, that is

$$
\left[f^{n}\left(a_{m} f^{m}+a_{m-1} f^{m-1}+\cdots+a_{1} f+a_{0}\right)\right]^{(k)}\left[g^{n}\left(a_{m} g^{m}+a_{m-1} g^{m-1}+\cdots+a_{1} g+a_{0}\right)\right]^{(k)} \equiv 1,
$$

then by Lemma 2.7 we can get a contradiction.

Hence, we deduce that $F \equiv G$, that is

$$
f^{n}\left(a_{m} f^{m}+a_{m-1} f^{m-1}+\cdots+a_{1} f+a_{0}\right)=g^{n}\left(a_{m} g^{m}+a_{m-1} g^{m-1}+\cdots+a_{1} g+a_{0}\right) .
$$

Let $h=\frac{f}{g}$. If $h$ is a constant, then substituting $f=g h$ in (3.8) we obtain

$$
a_{m} g^{n+m}\left(h^{n+m}-1\right)+a_{m-1} g^{n+m-1}\left(h^{n+m-1}-1\right)+\cdots+a_{0} g^{n}\left(h^{n}-1\right)=0,
$$

which implies $h^{d}=1$, where $d=(n+m, \ldots, n+m-i, \ldots, n), a_{m-1} \neq 0$ for some $i=0,1, \ldots, m$. Thus $f \equiv \operatorname{tg}$ for a constant $t$ such that $t^{d}=1$, where $d=(n+m, \ldots, n+m-i, \ldots, n), a_{m-i} \neq 0$ for some $i=0,1, \ldots, m$.

If $h$ is not a constant, then we know (3.8) that $f$ and $g$ satisfy the algebraic equation $R(f, g)=0$, where $R\left(\omega_{1}, \omega_{2}\right)=\omega_{1}^{n} P\left(\omega_{1}\right)-\omega_{2}^{n} P\left(\omega_{2}\right)$.

Case(ii) If $l=1$ and from (3.1) to (3.6) and also from Lemma 2.6, we get

$$
\begin{aligned}
\Delta_{2} & =(2 k+3) \Theta(\infty, f)+2 \Theta(\infty, g)+\Theta(0, f)+\Theta(0, g)+\delta_{k+1}(0, f)+\delta_{k+1}(0, g)+\delta_{k+2}(0, f) \\
& >(2 k+10)-\frac{5 k+13}{s(n+m)}
\end{aligned}
$$

Since $s(n+m)>5 k+13$, we get $\Delta_{2}>2 k+9$.

By continuing as in case(i), we get case(ii).

Case(iii) If $l=0$ and from (3.1) to (3.6) and also from Lemma 2.6, we get

$$
\begin{aligned}
\Delta_{3} & =(2 k+3) \Theta(\infty, f)+(2 k+4) \Theta(\infty, g)+\Theta(0, f)+\Theta(0, g)+2 \delta_{k+1}(0, f)+3 \delta_{k+1}(0, g) \\
& >(4 k+14)-\frac{9 k+16}{s(n+m)}
\end{aligned}
$$


Since $s(n+m)>9 k+16$, we get $\Delta_{2}>4 k+13$.

By continuing as in case(i), we get case(iii).

This completes the proof of Theorem 1.1.

Proof of Theorem 1.2. Since $f$ and $g$ are entire functions we have $\bar{N}(r, f)=\bar{N}(r, g)=0$. Proceeding as in the proof of Theorem 1.1 we can easily prove Theorem 1.2.

\section{References}

[1] M. L. Fang and X. H. Hua, Entire functions that share one value, J. Nanjing Univ. Math., biquartetly 13(1) (1996), 44-48.

[2] M. L. Fang, Uniqueness and value-sharing of entire functions, Comput. Math. Appl., 44(2002), 823-831.

[3] W. K. Hayman, Meromorphic Functions, Clarendon Press, Oxford, 1964.

[4] Lahiri, I. and Sarkar, A., Uniqueness of meromorphic functions and derivative, J. Inequal. Pure Appl. Math., 5(1) (2004), Art.20.

[5] H. Y. Xu and T. B. Cao, Uniqueness of entire or meromorphic functions sharing one value or a function with finite weight, J. Inequal. Pure Appl. Math., 10(3)(2009) Art.88.

[6] L. Yang, Value Distribution Theory, Springer Verlag, Berlin, 1993.

[7] H. X. Yi and C. C. Yang, Unicity Theory of Meromorphic Functions, Science Press, Beijing, 1995.

[8] C. C. Yang and X. H. Hua, Uniqueness and value-sharing of meromorphic functions, Ann. Acad. Sci. Fenn. Math., 22(2)(1997), 395-406.

[9] C. C. Yang and H. X. Yi, Uniqueness Theory of Meromorphic Functions, Science Press/ Kluwer Academic Publishers, Beijing/ New York, (1995/2003).

[10] X. Y. Zhang and W. C. Lin, Uniqueness and value-sharing of meromorphic functions, J. Math. Anal. Appl., 43(2)(2008), 938-950.

[11] X. Y. Zhang, J. F. Chen and W. C. Lin, Entire or meromorphic functions sharing one value, Comput. Math. Appl., 56(2008), 1876-1883.

[12] Q. C. Zhang, Meromorphic functions that shares one small function with its derivative, J. Inequal. Pure Appl. Math., 6(4)(2005) Art.116.

[13] T.Zhang and W. Lu, Uniqueness theorems on meromorphic functions sharing one value, Comput. Math. Appl., 55(2008), 2981-2992.

Department of Mathematics, Central College Campus, Bangalore University, Bangalore-560 001, India.

E-mail: pree.tam@rediffmail.com

Department of Mathematics, Central College Campus, Bangalore University, Bangalore-560 001, India.

E-mail: a.tanuja1@gmail.com 